\title{
Magnetic Field induced formation of magnetic wires into thin elastic membranes with controlled properties.
}

\author{
Daniela Lorenzo ${ }^{1, a}$, Despina Fragouli ${ }^{1, b}$, George C. Anyfantis ${ }^{1}$, \\ Roberto Cingolani ${ }^{2}$, Athanassia Athanassiou ${ }^{1,2}$ \\ ${ }^{1}$ Center for Biomolecular Nanotechnologies (CBN) @UNILE, Istituto Italiano di Tecnologia (IIT) Via \\ Barsanti, 73010 Arnesano (LE), Lecce, Italy \\ ${ }^{2}$ Istituto Italiano di Tecnologia (IIT) via Morego 30, 16163 Genova \\ adaniela.lorenzo@iit.it, bdespina.fragouli@iit.it
}

Keywords: Thin films, magnetic nanoparticles, nanocomposites, magnetic assembly.

\begin{abstract}
.
We present the fabrication of magnetic elastomeric membranes consisting of aligned superparamagnetic microwires embedded in a polymeric matrix. The wires are formed by the magnetic assembly of colloidal iron oxide nanoparticles (NPs) dispersed in the prepolymer matrix, during the curing of the polymer and the solvent evaporation. The appropriate combination of the NPs quantity and of the viscosity of the matrix results in the formation of thin membranes of about $10 \mu \mathrm{m}$, containing few layers of aligned wires which after the polymer curing are blocked in fixed position into the matrix. The orientation and the dimensions of the magnetic wires depend on the direction and intensity of the external MF respectively.
\end{abstract}

\section{Introduction}

Magnetic elastomers represent a new type of composites, consisting of small (mainly micron-sized) magnetic particles dispersed in a highly elastic polymeric matrix. This combination displays materials with novel and often enhanced properties [1]. In particular, the fabrication of elastomers with magnetic periodic structures, results in materials with anisotropically enhanced properties, where the physical characteristics of the elastomer such as its special mechanical properties, are combined with the anisotropic properties of the aligned structures [2].

Moreover numerous experimental and theoretical studies are conducted on the induced assembly of magnetic particles for the formation of wire like structures with controllable spacing between them, by varying the intensity of the applied magnetic field (MF). In particular, most of these studies are focused on the control of the distance between aligned columns of field-induced assembled NPs in ferrofluids. In such cases the NPs are aligned vertical to the sample surface. The length of these structures is specified by the cell thickness, while the structures are not immobilized into the cell. In fact when the MF is removed the structures lose their orientation [3].

In this study we focus in the formation of thin membranes of magnetic elastomers containing few layers of aligned superparamagnetic wires in parallel to the membranes surface direction. In particular it is performed a study on the wires formation in the bulk matrix, and on the variation of the wires orientation and dimensions, as a function of the MF direction and intensity respectively. The possibility of varying the dimensions of the wires in an horizontal plane, and the fixing of these wires in permanent positions into the elastomeric membranes, offers interesting prospects for the use of these materials for magneto-optical, and display devices, protective coatings, electromagnetic wave absorbers, and also in the use of such magnetic membranes, in microfluidic applications, or biological scaffolds. 


\section{Materials and Methods}

The polydimethylsiloxane (PDMS) elastomer was prepared from a commercial bi-component resin (Sylgard 184 Silicone Elastomer Kit, Dow Corning Corporation): the first component (base) contains a cross-linkable silicone polymer, and the second component is the curing agent and the hydrosilylation catalyst. The spherical colloidal NPs, used as a fillers, are spinel cubic $\gamma$ $\mathrm{Fe}_{2} \mathrm{O}_{3} / \mathrm{Fe}_{3} \mathrm{O}_{4}$ of $(10 \pm 1 \mathrm{~nm})$ diameter (Fig. 1), synthesized by modifying a surfactant-assisted nonaqueous synthetic approach $[4,5]$.

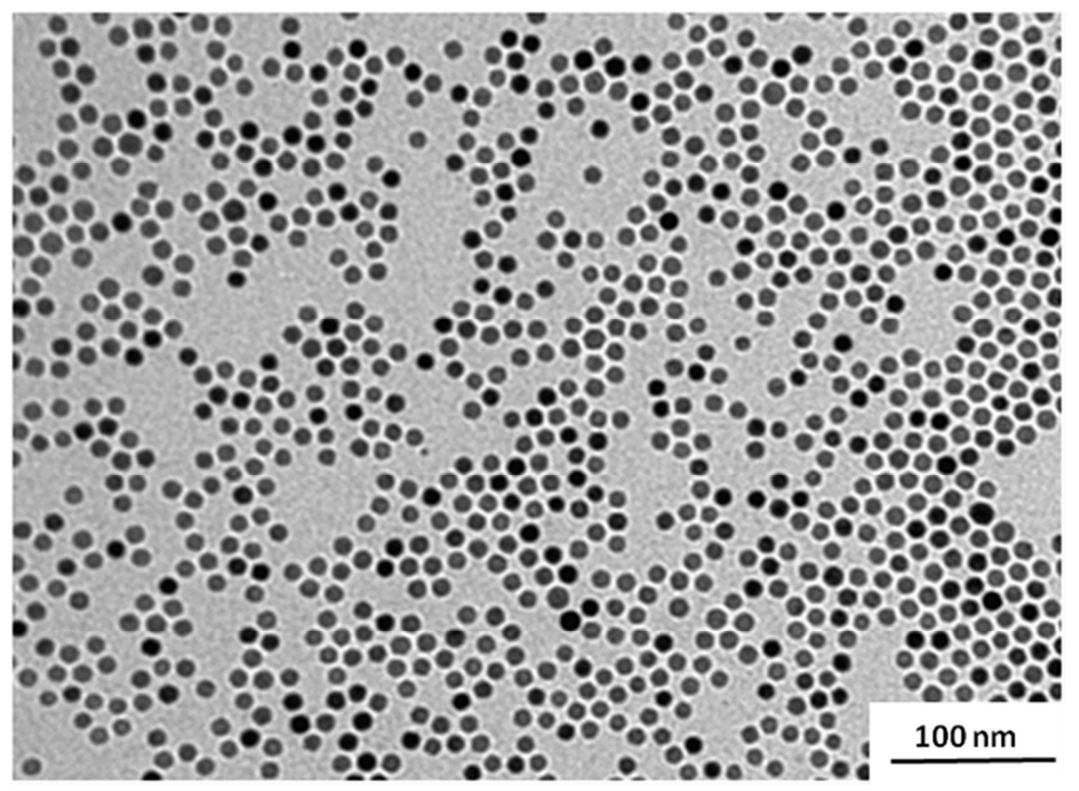

Figure 1: Transmission electron microscopy (TEM) image of the magnetic NPs with mean diameter of $10 \pm 1 \mathrm{~nm}$.

An amount of NPs dispersed in toluene solution, $(0.4 \%$ by weight with respect to the PDMS prepolymer) was mixed with a 1:1 solution of the PDMS base component and toluene before the curing. Subsequently the solution NPs/base component/solvent has been mixed in a 10:1 ratio with the curing agent (prepolymer:curing agent) and spin coated on a glass substrate.

The so obtained membrane of thickness c.a. $10 \mu \mathrm{m}$ was immediately exposed to a homogeneous external MF produced by an electromagnet and kept overnight under ambient conditions. In order to obtain the final solidification of the elastomeric matrix and the immobilization of the wires in defined positions, after alignment completion and removal of the MF the samples were cured by heating at $95^{\circ} \mathrm{C}$ for $4 \mathrm{~h}$. The experimental set-up is shown in Fig. 2.

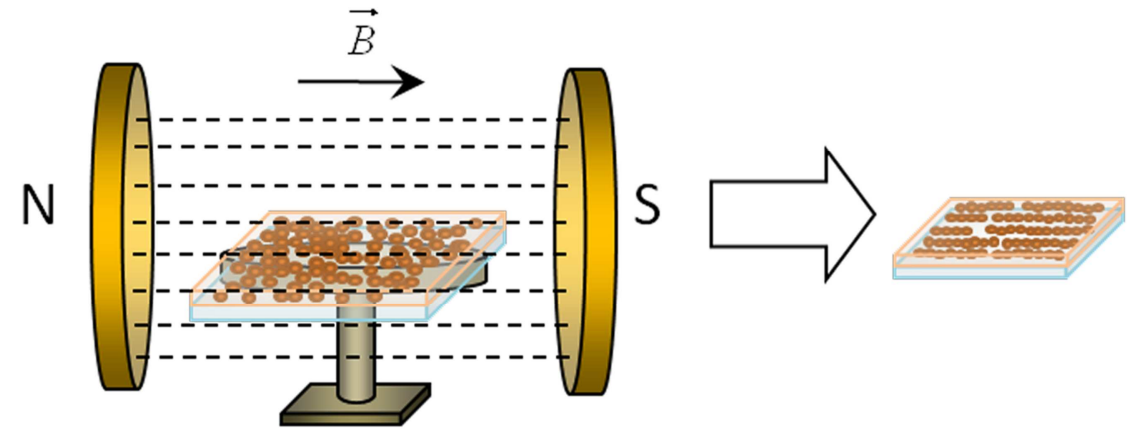

Figure 2: Schematic representation set-up of the alignment under MF

Images of the so formed wires have been acquired by using a digital camera (Carl Zeiss, AxioCam IC3) connected to a high magnification (40x) optical microscope (Carl Zeiss, Axio Scope A1). From the acquired pictures, the wires' length has been measured using a particle analysis system 
Image J software. A confocal microscope (Leica TCS-SP5 AOBS, Leica Application Suite Imaging Software) with high magnification in bright field (63X, 1.40 NA oil immersion objective and brightfield detector) was instead used to measure the width of the wires as a function of external MF.

\section{Results and discussion}

Fig. 3 shows the optical images of two membranes before and after the application of the external MF. As shown, initially the NPs form aggregated structures of irregular sizes in the polymeric matrix (Fig. 3a). On the other hand the application of an external MF causes the movement of the aggregated structures parallel to the MF direction, and their assembling for the formation of wirelike structures into the elastomeric membrane (Fig.3b) during the PDMS cross-linking reaction. These wires become quite long, and can ultimately extend over tens of microns. After the end of the PDMS polymerization reaction the wires are blocked in the specific positions and the membrane contains permanently aligned structures of defined shape and orientation. On the other hand, if the MF is removed after few minutes from the process of alignment, the aggregated structures slowly tend to re-disperse in the elastomeric matrix, since at this point the polymeric matrix has still low viscosity, and the Brownian motion of the NPs is sufficiently higher than the magnetic interactions that keep the superparamagnetic NPs together.

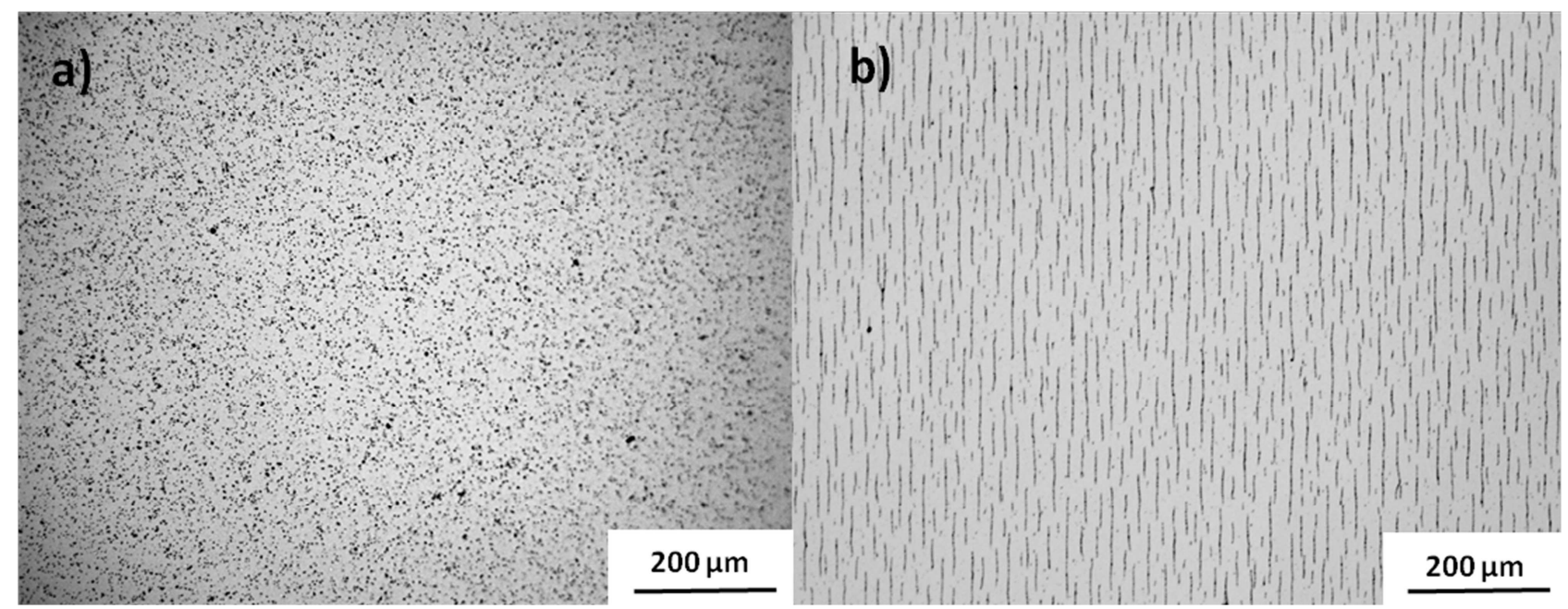

Figure 3: Microscope images of NPs/PDMS film before (a) and after (b) the application of an external MF (280 mT)

When instead of removing, the MF is rotated by $90^{\circ}$ with respect to its' initial direction, the aligned wires are decomposed and are now moving into the matrix forming new wires aligned parallel to the new MF direction. Fig. 4 shows the microscopy images of the kinetics of this process. In fact, immediately after the MF rotation the wires are not rotating but are breaking into smaller parts (Fig. 4b) while the wires of their origin can be easily identified. Thus, the initial clusters after the application of the MF for the first time are interacting with each other, in a small range, forming permanent elongated structures which after the rotation of the MF are moving into the matrix in order to form the new wires. Indeed Fig. $4 \mathrm{c}$, and $4 \mathrm{~d}$ show that these clusters are moving into the matrix and are assembling with the neighboring structures in order to form new wires with direction parallel to the new MF. 

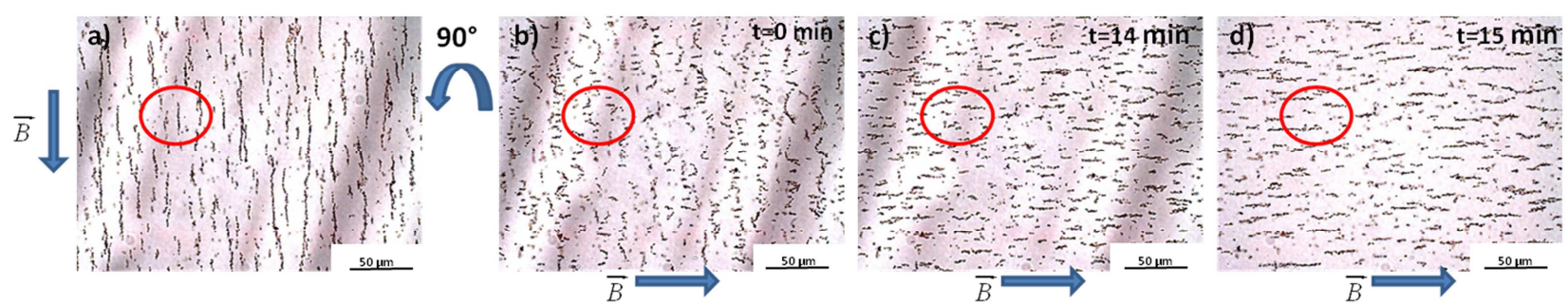

Figure 4: Effect of MF rotation on the wires. (a) Optical image after the formation and the alignment of the wires in the PDMS membrane after 60min under MF. (b), (c), (d) Pictures sequence of the wires rotation and assembling after the rotation of the MF by $90^{\circ}$ with respect to the initial direction. Time 0 min indicates the time immediately after the MF rotation.

Under a stable MF direction, the wires are formed, and after the polymer curing they are immobilized in specific positions into the matrix, even if the MF is removed. The dimensions of the final wire structures strongly depend on the MF intensity. In fact Fig. 5 shows the dependence of the width and the length of the wires as a function of the MF intensity. At low MF the wires are shorter and thicker while as the MF increases they tend to stretch and become narrower. Specifically the width of the wires decreases from about 1.1 to $0.8 \mu \mathrm{m}$ for increasing MF (from 70 to $200 \mathrm{mT}$ ) while their length increases from c.a. 80 to $200 \mu \mathrm{m}$ (Fig. 5b). In fact at low MF intensities, the developed magnetic forces between clusters is not enough in order to move them into the matrix sufficiently fast before the increase of the viscosity of the polymer. Therefore these clusters form small elongated structures, dispersed in the film. On the contrary, when stronger MFs are applied, the induced interaction force between neighbor clusters dramatically raises. In this way the clusters can be easily moved into the matrix rapidly, and longer wires are obtained.

a)

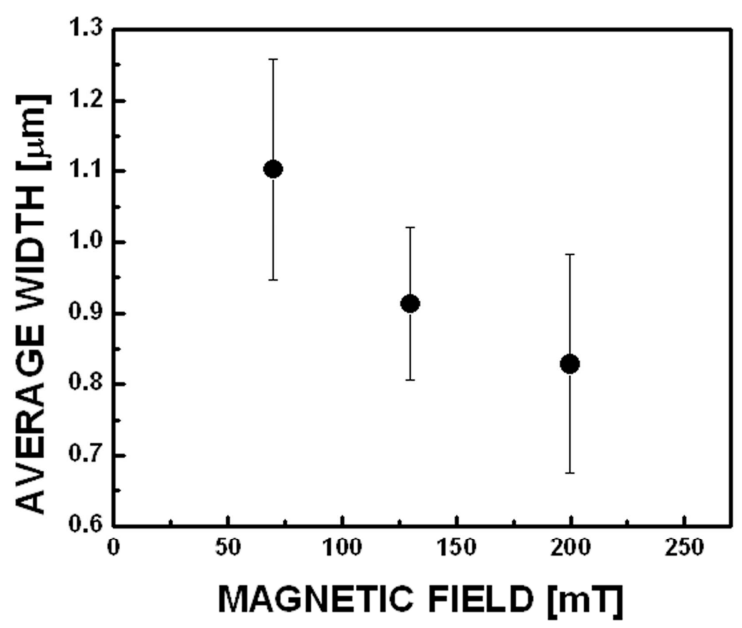

b)

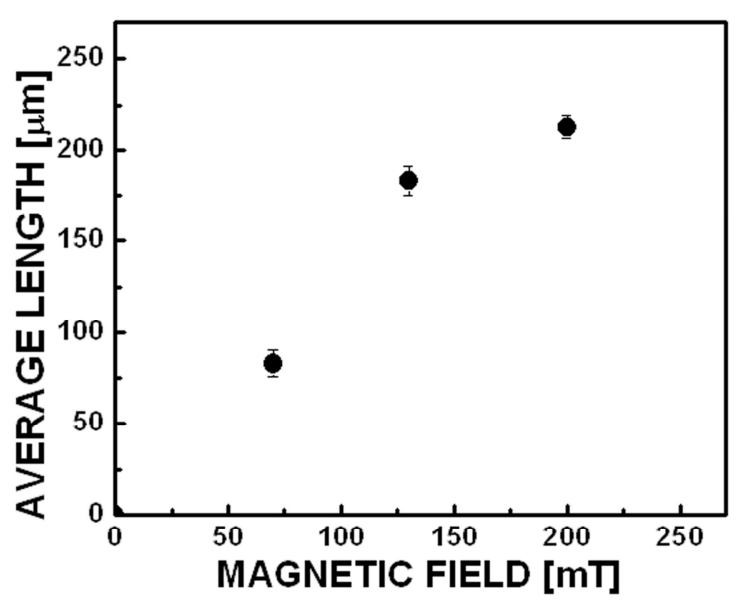

Figure 5: Dependence of average width (a) and average length (b) of the wires as a function of MF intensity.

\section{CONCLUSIONS}

In this study superparamagnetic elastomeric membranes have been obtained, by the field induced assembly of iron oxide NPs. The membranes contain few layers of magnetic wires aligned in a direction parallel to the MF and immobilized in these defined positions. By changing the MF direction and intensity we were able to control and manipulate the wires' orientation and dimensions into the matrix. This ability to control the distribution of wires into elastomers gives the opportunity to develop multifunctional composite material ready to be used in various applications. 


\section{References}

[1] D. Fragouli, R. Buonsanti, G. Bertoni, C. Sangregorio, C. Innocenti, A. Falqui, D. Gatteschi, P.D. Cozzoli, A. Athanassiou, R. Cingolani, Dynamical Formation of Spatially Localized Arrays of Aligned Nanowires in Plastic Films with Magnetic Anisotropy, ACS Nano 4 (2010) 1873-1878.

[2] Z. Varga, G. Filipcsei, M. Zrínyi, MF Sensitive Functional Elastomers with Tuneable Elastic Modulus, Polym. 47 (2006) 227-233.

[3] S. Y. Yang, H. E. Horng, C.-Y. Hong, H. C. Yang, M. C. Chou, C. T. Pan, Y. H. Chao, Control Method for the Tunable Ordered Structures in Magnetic Fluid Microstrips, J. Appl. Phys. 93 (2003) 3457-3460.

[4] T. Hyeon, S. S. Lee, J. Park, Y. Chung, H. B. Na, Synthesis of Highly Crystalline and Monodisperse Maghemite Nanocrystallites without a Size-Selection Process, J. Am. Chem. Soc. 123 (2001) 12798-12801.

[5] J. Park, E. Lee, N. M. Hwang, M. Kang, S. C. Kim, Y. Hwang, J. G. Park, H. J. Noh, J. Y. Kim, J. H. Park, T.Hyeon, One-Nanometer-Scale Size-Controlled Synthesis of Monodisperse Magnetic Iron Oxide Nanoparticles, Angew. Chem. Int. Ed. 44 (2005) 2872-2877. 
Adaptive, Active and Multifunctional Smart Materials Systems

10.4028/www.scientific.net/AST.77

Magnetic Field Induced Formation of Magnetic Wires into Thin Elastic Membranes with Controlled Properties

10.4028/www.scientific.net/AST.77.343 